Copyright $(2008$ Institute of Electrical and Electronics Engineers, Inc.

All rights reserved.

Personal use of this material, including one hard copy reproduction, is permitted.

Permission to reprint, republish and/or distribute this material in whole or in part for any other purposes must be obtained from the IEEE.

For information on obtaining permission, send an e-mail message to stds-ipr@ieee.org.

By choosing to view this document, you agree to all provisions of the copyright laws protecting it.

Individual documents posted on this site may carry slightly different copyright restrictions.

For specific document information, check the copyright notice at the beginning of each document. 


\title{
On Designing Time-Varying Delay Feedback Controllers for Master-Slave Synchronization of Lur'e Systems
}

\author{
Qing-Long Han
}

\begin{abstract}
This paper is concerned with the problem of designing time-varying delay feedback controllers for master-slave synchronization of Lur'e systems. Two cases of time-varying delays are fully considered; one is the time-varying delay being continuous uniformly bounded while the other is the time-varying delay being differentiable uniformly bounded with the derivative of the delay bounded by a constant. Based on Lyapunov-Krasovskii functional approach, some delay-dependent synchronization criteria are first obtained and formulated in the form of linear matrix inequalities (LMIs). The relationship between synchronization criteria for the two cases of time-varying delays is built. Then, sufficient conditions on the existence of a time-varying delay feedback controller are derived by employing these newly-obtained synchronization criteria. The controller gains can be achieved by solving a set of LMIs. Finally, Chua's circuit is used to illustrate the effectiveness of the design method.
\end{abstract}

Index Terms -Absolute stability, controller design, linear matrix inequality (LMI), Lur'e systems, state feedback, static output feedback, synchronization, time-varying delay.

\section{INTRODUCTION}

C HAOTIC synchronization [2] has received considerable attention due to its practical applications such as secure communications in which an information bearing signal is hidden on a chaotic carrier signal [10], [12], [22]. As is well known, there are some nonlinear systems, such as Chua's circuit, $n$-scroll attractors and hyperchaotic attractors [23], which can be represented as Lur'e systems. Therefore, master-slave synchronization for Lur'e systems has well studied in the last decade [4], [5], [14], [16], [17], [19], [21], [22]. For the master-slave systems which are identical and autonomous, Curran and Chua [4] built the relationship between a synchronization problem and absolute stability theory. Curran et al. [5] extended the work in [4] using a Lur'e-Postnikov Lyapunov functional. The main idea in [4], [5] is to employ a unified approach [22], which reformulates chaotic synchronization as a Lur'e system and then discusses the absolute stability of its error system. For the master-slave systems which are identical and nonautonomous, the synchronization scheme was interpreted as a model-reference control scheme in standard plant

Manuscript received June 4, 2006; revised December 30, 2006. This work was supported in part by Central Queensland University for the Research Advancement Awards Scheme Project "Robust Fault Detection, Filtering and Control for Uncertain Systems with Time-Varying Delay". This paper was recommended by Associate Editor C. Hadjicostis.

The author is with the School of Computing Sciences, Central Queensland University, Rockhampton QLD 4702, Australia (e-mail: q.han@cqu.edu.au).

Digital Object Identifier 10.1109/TCSI.2007.899627 form, with exogenous input and regulated output [18]. For the master-slave systems which are nonidentical, i.e., parameters are mismatch between the two systems, the reader is referred to [19], [20] and the references therein.

Due to the propagation delay frequently encountered in remote master-slave synchronization schemes, recently, there have been some research efforts to investigate the delay effect on master-slave synchronization. For example, in [23], some delay-independent and delay-dependent criteria were derived for master-slave synchronization of Lur'e systems using a constant time-delay static error output feedback control. In order to handle the case where the master-slave systems can not be synchronized by a pure time-delay static error output feedback control, Liao and Chen [13] considered the feedback control including both the current error state feedback and the delayed static error output feedback, and gave some simple algebraic conditions which are easy to be verified. In [1], the results in [13], [23] were generalized and improved. Huang et al. [11] extended the setting in [13] from a constant time-delay feedback control to a time-varying delay one and derived some delay-independent and delay-dependent synchronization criteria under the assumption that the bound of time-derivative of the time-varying delay is less than one. However, when deriving delay-dependent sufficient conditions for master-slave synchronization, both model transformation [11], [13], [23] and bounding technique for cross terms [11] were employed. As pointed out by Gu et al. [7], model transformation sometimes will induce additional dynamics. Although a tighter bounding for cross terms can reduce the conservatism, however, there is no obvious way to obtain a much tighter bounding for cross terms. To sum up, in order to derive a much less conservative synchronization condition, we are in a position to avoid using both model transformation and bounding technique for cross terms, which is the first motivation of the present study.

It should be pointed out that the results in [1], [11], [13], [23] were only concerned with deriving some sufficient conditions for master-slave synchronization of Lur'e systems, and did not address how to design the controller. A nonlinear optimization approach proposed by Suykens and Vandewalle [16] was suggested to handle the controller design issue. However, the nonlinear optimization problem was a nonconvex one. Non-differentiability might occur [16]. How to easily design the controller using a convex optimization problem is the second motivation of the current study.

In this paper, we will deal with the problem of master-slave synchronization of Lur'e systems using time-varying delay 
feedback control. Two cases of the time-varying delays will be studied and some delay-dependent synchronization criteria will be derived without employing any model transformation and bounding technique for cross terms. We will build the relationship between the synchronization criteria for the two cases of time-varying delays. Based on the synchronization criteria, we will give some sufficient conditions on the existence of a time-varying delay error feedback controller. These sufficient conditions will be formulated in the form of linear matrix inequalities (LMIs). Instead of solving a nonlinear optimization problem, we will design the controller by solving a set of LMIs. We will use Chua's circuit to illustrate the effectiveness of the design method.

Notation: $\mathbb{R}^{n}$ denotes the $n$-dimensional Euclidean space. $\mathbb{R}^{m \times n}$ is the set of all $m \times n$ real matrices. For symmetric matrices $P$ and $Q$, the notation $P>Q$ (respectively, $P \geq Q$ ) means that matrix $P-Q$ is positive definite (respectively, positive semi-definite). $I$ is an identity matrix of appropriate dimensions. $\operatorname{tr}(W)$ denotes the trace of matrix $W$. $\operatorname{diag}\left(a_{1}, a_{2}, \cdots, a_{n}\right)$ denotes the block-diagonal matrix. For an arbitrary matrix $W$ and two symmetric matrices $P$ and $Q$, the symmetric term in a symmetric matrix is denoted by $*$, i.e.,

$$
\left(\begin{array}{cc}
P & W \\
* & Q
\end{array}\right)=\left(\begin{array}{cc}
P & W \\
W^{T} & Q
\end{array}\right)
$$

\section{Problem Statement}

Consider a general master-slave synchronization scheme using time-varying delay static error feedback control

$$
\begin{aligned}
& \mathcal{M}:\left\{\begin{array}{l}
\dot{x}(t)=A x(t)+B \varphi(C x(t)) \\
z_{x}(t)=H x(t)
\end{array}\right. \\
& \mathcal{S}:\left\{\begin{array}{l}
\dot{y}(t)=A y(t)+B \varphi(C y(t))+u(t) \\
z_{y}(t)=H y(t)
\end{array}\right. \\
& \mathcal{U}: \begin{aligned}
u(t)=-K(x(t)-y(t)) \\
\quad+L\left(z_{x}(t-r(t))-z_{y}(t-r(t))\right)
\end{aligned}
\end{aligned}
$$

with master system $\mathcal{M}$, slave system $\mathcal{S}$ and controller $\mathcal{C}$, where the time delay $r(t)$ satisfying $0 \leq r(t) \leq r_{M}$ is a time-varying function. The master and slave systems are Lur'e systems with state vectors $x(t), y(t) \in \mathbb{R}^{n}$, and the output vectors $z_{x}(t), z_{y}(t) \in \mathbb{R}^{l}$, respectively; $A \in \mathbb{R}^{n \times n}, B \in \mathbb{R}^{n \times m}$, $C \in \mathbb{R}^{m \times n} H \in \mathbb{R}^{l \times n}$ are constant matrices; $\varphi(\cdot): \mathbb{R}^{m} \rightarrow \mathbb{R}^{m}$ is a memoryless nonlinear vector valued function which is globally Lipschitz $\varphi(0)=0$, and suppose that the nonlinearity $\varphi(\cdot)$ is time-invariant, decoupled, and satisfies a sector condition with $\varphi_{i}(\xi)$ belonging to a sector $[0, k]$, i.e.,

$$
\varphi_{i}(\xi)\left[\varphi_{i}(\xi)-k \xi\right] \leq 0 \quad \forall t \geq 0 \quad \forall \xi \in \mathbb{R} .
$$

Defining a signal $e(t)=x(t)-y(t)$, we have the error system

$$
\dot{e}(t)=(A+K) e(t)+M e(t-r(t))+B \eta(C e(t), y(t))
$$

where $M=-L H$ and $\eta(C e(t), y(t))=\varphi(C e(t)+$ $C y(t))-\varphi(C y(t))$. Let $C=\left[c_{1}, c_{2}, \cdots, c_{m}\right]^{T}, c_{i} \in \mathbb{R}^{n}$, $i=1,2, \cdots, m$. Suppose that $\eta(C e(t), y(t))$ belongs to the sector $[0, k]$ (Curran and Chua [4]; Suykens and Vandewalle [16]), i.e., for $\forall t \geq 0, \forall e(t), y(t)$

$$
\eta_{i}\left(c_{i}^{T} e(t), y(t)\right)\left[\eta_{i}\left(c_{i}^{T} e(t), y(t)\right)-k c_{i}^{T} e(t)\right] \leq 0
$$

The initial condition of system (5) is defined as

$$
e(\theta)=\phi(\theta) \quad \forall \theta \in\left[-r_{M}, 0\right]
$$

where $\phi(\theta)$ is a continuous vector valued function.

The purpose of this paper is to design the time-varying delay controller (3), i.e., to find the controller gains $K$ and $L$, such that the system described by (5)-(7) is globally asymptotically stable, which means that the system described by (1)-(3) synchronizes.

Throughout this paper, we will handle the following two cases of the time-varying delay $r(t)$.

Case 1) $r(t)$ is a continuous function satisfying

$$
0 \leq r(t) \leq r_{M}<\infty \quad \forall t \geq 0 .
$$

Case 2) $r(t)$ is a differentiable function satisfying

$$
0 \leq r(t) \leq r_{M}<\infty, \quad \dot{r}(t) \leq r_{d}<\infty \quad \forall \quad t \geq 0 .
$$

In the above, $r_{M}$ and $r_{d}$ are constants.

Remark 1: One can clearly see that Case 1 includes Case 2 as a special case. Case 1 only requires that the time-varying delay is a bounded continuous function while Case 2 needs additional information regarding the bound of the derivative of the timevarying delay. If the time-varying delay is differentiable and $r_{d}<1$, one can get a less conservative result using Case 2 than that employing Case 1. However, if the time-varying delay is not differentiable for all $t \geq 0$, only Case 1 can be used to handle the situation.

The following lemma is useful in deriving synchronization criteria.

Lemma 1: For any constant matrix $W \in \mathbb{R}^{n \times n}, W=W^{T}>$ 0 , scalar $0 \leq r(t) \leq r_{M}$, and vector function $\dot{e}:\left[-r_{M}, 0\right] \rightarrow$ $\mathbb{R}^{n}$ such that the following integration is well defined, then

$$
\begin{aligned}
& -r_{M} \int_{-r(t)}^{0} \dot{e}^{T}(t+\xi) W \dot{e}(t+\xi) d \xi \leq\left(e^{T}(t) \quad e^{T}(t-r(t))\right) \\
& \times\left(\begin{array}{cc}
-W & W \\
W & -W
\end{array}\right)\left(\begin{array}{c}
e(t) \\
e(t-r(t))
\end{array}\right) .
\end{aligned}
$$

Proof: Use Lemma 1 in [9] to obtain

$$
\begin{aligned}
r_{M} & \int_{-r(t)}^{0} \dot{e}^{T}(t+\xi) W \dot{e}(t+\xi) d \xi \\
& \geq\left(\int_{-r(t)}^{0} \dot{e}(t+\xi) d \xi\right)^{T} W\left(\int_{-r(t)}^{0} \dot{e}(t+\xi) d \xi\right) \\
& =[e(t)-e(t-r(t))]^{T} W[e(t)-e(t-r(t))] .
\end{aligned}
$$

Re-arranging some terms yields (10). 


\section{STABILITY ANALYSIS}

For Case 1, choosing a Lyapunov-Krasovskii functional candidate as

$V\left(t, e_{t}\right)=e^{T}(t) P e(t)+\int_{t-r_{M}}^{t}\left(r_{M}-t+\xi\right) \dot{e}^{T}(\xi)\left(r_{M} R\right) \dot{e}(\xi) d \xi$

where $e_{t}$ is defined as $e_{t}=e(t+\theta), \forall \theta \in\left[-r_{M}, 0\right]$, and $P \in \mathbb{R}^{n \times n}, P=P^{T}>0 ; R \in \mathbb{R}^{n \times n}, R=R^{T}>0$, we have the following result.

Proposition 1: Under Case 1, the error system described by (5)-(7) is globally asymptotically stable if there exist $n \times n$ real matrices $P=P^{T}>0, R=R^{T}>0$, and $\Lambda=\operatorname{diag}\left(\lambda_{1}, \lambda_{2}, \cdots, \lambda_{m}\right)>0$ such that

$$
\Psi^{(1)}=\left(\begin{array}{cccc}
(1,1) & P M+R & (1,3) & (1,4) \\
* & -R & 0 & (2,4) \\
* & * & -2 \Lambda & (3,4) \\
* & * & * & -R
\end{array}\right)<0
$$

where

$$
\begin{aligned}
& (1,1)=(A+K)^{T} P+P(A+K)-R \\
& (1,3)=P B+k C^{T} \Lambda \\
& (1,4)=r_{M}(A+K)^{T} R \\
& (2,4)=r_{M} M^{T} R \\
& (3,4)=r_{M} B^{T} R .
\end{aligned}
$$

Proof: Taking the derivative of $V\left(t, e_{t}\right)$ with respect to $t$ along the trajectory of (5) yields

$$
\begin{aligned}
\dot{V}\left(t, e_{t}\right)= & e^{T}(t)\left[(A+K)^{T} P+P(A+K)\right] e(t) \\
& +2 e^{T}(t) P M e(t-r(t))+2 e^{T}(t) P B \eta(C e(t), y(t)) \\
& +\dot{e}^{T}(t)\left(r_{M}^{2} R\right) \dot{e}(t)-\int_{t-r_{M}}^{t} \dot{e}^{T}(\xi)\left(r_{M} R\right) \dot{e}(\xi) d \xi .
\end{aligned}
$$

From (6), for $\Lambda=\operatorname{diag}\left(\lambda_{1}, \lambda_{2}, \cdots, \lambda_{m}\right)>0$, we have

$$
\begin{aligned}
\dot{V}\left(t, e_{t}\right) \leq & e^{T}(t)\left[(A+K)^{T} P+P(A+K)\right] e(t) \\
& +2 e^{T}(t) P M e(t-r(t)) \\
& +2 e^{T}(t) P B \eta(C e(t), y(t))+\dot{e}^{T}(t)\left(r_{M}^{2} R\right) \dot{e}(t) \\
& -\int_{t-r_{M}}^{t} \dot{e}^{T}(\xi)\left(r_{M} R\right) \dot{e}(\xi) d \xi-2 \eta^{T}(C e(t), y(t)) \\
& \times \Lambda \eta(C e(t), y(t))+2 k \eta^{T}(C e(t), y(t)) \Lambda C e(t) .
\end{aligned}
$$

Use Lemma 1 to obtain

$$
\begin{aligned}
& -\int_{t-r_{M}}^{t} \dot{e}^{T}(\xi)\left(r_{M} R\right) \dot{e}(\xi) d \xi \leq\left(e^{T}(t) \quad e^{T}(t-r(t))\right) \\
& \times\left(\begin{array}{cc}
-R & R \\
R & -R
\end{array}\right)\left(\begin{array}{c}
e(t) \\
e(t-r(t))
\end{array}\right) .
\end{aligned}
$$

Noting that (5) is true, the following holds:

$$
\dot{e}^{T}(t)\left(r_{M}^{2} R\right) \dot{e}(t)
$$

$$
=q^{T}(t)\left(\begin{array}{c}
(A+K)^{T} \\
M^{T} \\
B^{T}
\end{array}\right)\left(r_{M}^{2} R\right)((A+K) M B) q(t)
$$

where

$$
q^{T}(t)=\left(e^{T}(t) \quad e^{T}(t-r(t)) \quad \eta^{T}(C e(t), y(t))\right) .
$$

Then, we have

$$
\dot{V}\left(t, e_{t}\right) \leq q^{T}(t) \Xi q(t)
$$

where

$$
\Xi=\left(\begin{array}{ccc}
\Xi_{11} & \Xi_{12} & \Xi_{13} \\
* & \Xi_{22} & \Xi_{23} \\
* & * & \Xi_{33}
\end{array}\right)
$$

with

$$
\begin{aligned}
\Xi_{11}= & (A+K)^{T} P+P(A+K) \\
& -R+(A+K)^{T}\left(r_{M}^{2} R\right)(A+K) \\
\Xi_{12}= & P M+R+(A+K)^{T}\left(r_{M}^{2} R\right) M \\
\Xi_{13}= & P B+k C^{T} \Lambda+(A+K)^{T}\left(r_{M}^{2} R\right) B \\
\Xi_{22}= & -R+M^{T}\left(r_{M}^{2} R\right) M \\
\Xi_{23}= & M^{T}\left(r_{M}^{2} R\right) B \\
\Xi_{33}= & -2 \Lambda+B^{T}\left(r_{M}^{2} R\right) B .
\end{aligned}
$$

If $\Xi<0$, then there exists a sufficiently small $\varepsilon>0$ such that $\dot{V}\left(t, e_{t}\right) \leq q^{T}(t) \Xi q(t) \leq-\varepsilon q^{T}(t) q(t)<0$ for $q(t) \neq 0$, which means that the system described by (5)-(7) is globally asymptotically stable. In view of Schur complement, $\Xi<0$ is implied by $\Psi^{(1)}<0$. This completes the proof.

For Case 2, since $r(t)$ is a differentiable function, by making use of this additional information, we choose a Lyapunov-Krasovskii functional candidate as

$$
\tilde{V}\left(t, e_{t}\right)=V\left(t, e_{t}\right)+V_{3}\left(t, e_{t}\right)
$$

where $V\left(t, e_{t}\right)$ is defined in (11) and

$$
V_{3}\left(t, e_{t}\right)=\int_{t-r(t)}^{t} e^{T}(\xi) Q e(\xi) d \xi
$$

with $Q \in \mathbb{R}^{n \times n}, Q=Q^{T}>0$. Then, similar to the proof of Proposition 1, we can conclude the following result.

Proposition 2: Under Case 2, the error system described by (5)-(7) is globally asymptotically stable if there exist $n \times n$ real matrices $P=P^{T}>0, Q=Q^{T}>0, R=R^{T}>0$, and $\Lambda=\operatorname{diag}\left(\lambda_{1}, \lambda_{2}, \cdots, \lambda_{m}\right)>0$ such that

$$
\Psi^{(2)}=\Psi^{(1)}+\operatorname{diag}\left(Q,-\left(1-r_{d}\right) Q, 0,0\right)<0
$$

where $\Psi^{(1)}$ is defined in (12).

Remark 2: Employing model transformation and bounding technique for cross terms, Huang et al. [11] also considered the problem of global asymptotic stability for the error system described by (5)-(7), where the assumption that $\dot{r}(t) \leq r_{d}<1$ on time-varying delay $r(t)$ is required. However, from the proof process of Proposition 1, one can clearly see that neither model transformation nor bounding technique for cross terms is involved. Therefore, the global asymptotic stability criteria are expected to be less conservative. Moreover, the restriction $r_{d}<1$ is removed, which means that a fast time-varying delay is allowed. 
It is easy to see that Propositions 1 and 2 provide delay-dependent sufficient conditions for Cases 1 and 2, respectively, which can guarantee global asymptotic stability of the error system described by (5)-(7). Depending on the information of delay $r(t)$, we can decide to use Proposition 1 or Proposition 2. If $r(t)$ is a continuous function which is not differentiable for all $t \geq 0$, i.e., only the information about $r_{M}$ is available, we can only use Proposition 1. If $r(t)$ is a differentiable function, both Propositions 1 and 2 can be applied. The natural question is: Is there any relationship between Propositions 1 and 2? The following proposition well answers this question.

Proposition 3: Suppose that the delay $r(t)$ satisfies (9). Then, we have the following facts.

(i) For $r_{d} \geq 1$, there exist $n \times n$ real matrices $P=P^{T}>0, Q=Q^{T}>0, R=R^{T}>0$, and $\Lambda=\operatorname{diag}\left(\lambda_{1}, \lambda_{2}, \cdots, \lambda_{m}\right)>0$ such that $\Psi^{(2)}<0$ if and only if there exist $n \times n$ real matrices $P=P^{T}>0$, $R=R^{T}>0$, and $\Lambda=\operatorname{diag}\left(\lambda_{1}, \lambda_{2}, \cdots, \lambda_{m}\right)>0$ such that $\Psi^{(1)}<0$.

(ii) For $r_{d}<1$, if there exist $n \times n$ real matrices $P=P^{T}>0$, $R=R^{T}>0$, and $\Lambda=\operatorname{diag}\left(\lambda_{1}, \lambda_{2}, \cdots, \lambda_{m}\right)>0$ such that $\Psi^{(1)}<0$, then there exist $n \times n$ real matrices $P=P^{T}>0, Q=Q^{T}>0, R=R^{T}>0$, and $\Lambda=\operatorname{diag}\left(\lambda_{1}, \lambda_{2}, \cdots, \lambda_{m}\right)>0$ such that $\Psi^{(2)}<0$; However, the reverse is not necessarily true.

Proof: (i) Necessity is obvious. For sufficiency, if there exist $n \times n$ real matrices $P=P^{T}>0, R=R^{T}>0$, and $\Lambda=\operatorname{diag}\left(\lambda_{1}, \lambda_{2}, \cdots, \lambda_{m}\right)>0$ such that $\Psi^{(1)}<0$, then there exists a sufficiently small scalar $q_{0}>0$ such that

$$
\Psi^{(1)}+q_{0} I<0 .
$$

Choosing $q_{1}>0$ such that $\max \left\{q_{1},-\left(1-r_{d}\right) q_{1}\right\} \leq q_{0}$, we have

$$
\Psi^{(1)}+\operatorname{diag}\left(q_{1} I,-\left(1-r_{d}\right) q_{1} I, 0,0\right) \leq \Psi^{(1)}+q_{0} I .
$$

Letting $Q=q_{1} I$, combining (15) with (16) yield $\Psi^{(2)}<0$.

(ii) Similar to the proof of the sufficiency part in (i), we can conclude that for $r_{d}<1$, if there exist $n \times n$ real matrices $P=P^{T}>0, R=R^{T}>0$, and $\Lambda=\operatorname{diag}\left(\lambda_{1}, \lambda_{2}, \cdots, \lambda_{m}\right)>$ 0 such that $\Psi^{(1)}<0$, then there exist $n \times n$ real matrices $P=P^{T}>0, Q=Q^{T}>0, R=R^{T}>0$, and $\Lambda=$ $\operatorname{diag}\left(\lambda_{1}, \lambda_{2}, \cdots, \lambda_{m}\right)>0$ such that $\Psi^{(2)}<0$. In the following, we consider the reverse. Without loss of generality since there always exists an orthogonal transformation such that a real symmetric matrix can be transformed into a diagonal one, we assume that

$$
\Psi^{(1)}=\operatorname{diag}\left(\mu_{1} I, \mu_{2} I, \mu_{3} I, \mu_{4} I\right)
$$

where $\mu_{1} I \in \mathbb{R}^{n \times n}, \mu_{2} I \in \mathbb{R}^{n \times n}, \mu_{3} I \in \mathbb{R}^{m \times m}, \mu_{4} I \in \mathbb{R}^{n \times n}$ and $\mu_{j}(j=1,2,3,4)$ are real scalars satisfying

$$
\mu_{1}<0, \quad 0<\mu_{2}<-\left(1-r_{d}\right) \mu_{1}, \quad \mu_{j}<0(j=3,4) .
$$

It is easy to see that $\Psi^{(1)}$ is not negative definite. However, there exists an $n \times n$ real matrix $Q=q I>0$, where $0<-(1-$ $\left.r_{d}\right)^{-1} \mu_{2}<q<-\mu_{1}$, such that

$$
\Psi^{(1)}+\operatorname{diag}\left(Q,-\left(1-r_{d}\right) Q, 0,0\right)<0
$$

i.e. $\Psi^{(2)}<0$, which means that for this situation, the reverse is not true. This completes the proof.

Remark 3: The second statement "However, the reverse is not necessarily true" in Proposition 3 (ii) means that for some situation, for $r_{d}<1$, even if there exist $n \times n$ real matrices $P=P^{T}>0, Q=Q^{T}>0, R=R^{T}>0$, and $\Lambda=$ $\operatorname{diag}\left(\lambda_{1}, \lambda_{2}, \cdots, \lambda_{m}\right)>0$ such that $\Psi^{(2)}<0$, for the same $P=P^{T}>0, R=R^{T}>0$, and $\Lambda=\operatorname{diag}\left(\lambda_{1}, \lambda_{2}, \cdots, \lambda_{m}\right)>$ 0 , the inequality $\Psi^{(1)}<0$ is no longer satisfied.

Remark 4: If we consider the pure time-varying delay static error output feedback controller, then the error system (5) becomes

$$
\dot{e}(t)=A e(t)+M e(t-r(t))+B \varphi(C e(t), y(t)) .
$$

The corresponding stability conditions are easily derived by setting $K=0$ in (12) and (14) for Cases 1 and 2, respectively.

Remark 5: If there exist parameter perturbations in system's matrices, then we have the following uncertain master and slave systems

$$
\mathcal{M}:\left\{\begin{aligned}
\dot{x}(t)= & \left(A+E F(t) G_{0}\right) x(t) \\
& +\left(B+E F(t) G_{1}\right) \varphi(C x(t)) \\
z_{x}(t)= & H x(t)
\end{aligned}\right.
$$

and

$$
\mathcal{S}:\left\{\begin{aligned}
\dot{y}(t)= & \left(A+E F(t) G_{0}\right) x(t) \\
& +\left(B+E F(t) G_{1}\right) \varphi(C y(t))+u(t) \\
z_{y}(t)= & H y(t)
\end{aligned}\right.
$$

where $E, G_{0}$, and $G_{1}$ are known real constant matrices of appropriate dimensions, and $F(t)$ is an unknown continuous timevarying matrix function satisfying

$$
F^{T}(t) F(t) \leq I
$$

The corresponding error system becomes

$\dot{e}(t)=\left(A+E F(t) G_{0}+K\right) e(t)+M e(t-r(t))$

$$
+\left(B+E F(t) G_{1}\right) \eta(C e(t), y(t)) .
$$

Using the routine method of handling norm-bounded uncertainty [8], by Propositions 1 and 2, one can easily obtain more general results.

\section{Controller Design}

In this section, based on the analysis results in last section, we are in a position to address the issue of controller design. Applying Proposition 1 we first state and establish the following result for Case 1.

Proposition 4: Under Case 1, for a given scalar $\alpha>0$, the system described by (1)-(3) synchronizes, with the error system described by (5)-(7) having a unique and globally asymptotically stable equilibrium point $e(t)=0$, if there exist $n \times$ $n$ real matrices $\tilde{P}=\tilde{P}^{T}>0, \tilde{R}=\tilde{R}^{T}>0$, and $\tilde{\Lambda}=$ $\operatorname{diag}\left(\tilde{\lambda}_{1}, \tilde{\lambda}_{2}, \cdots, \tilde{\lambda}_{m}\right)>0, X, Y$ of appropriate dimensions such that

$$
\left(\begin{array}{ccccc}
(1,1) & Y+\tilde{P} & (1,3) & (1,4) & \alpha \tilde{R} \\
* & -\tilde{R} & 0 & (2,4) & 0 \\
* & * & -2 \tilde{\Lambda} & (3,4) & 0 \\
* & * & * & -\tilde{R} & 0 \\
* & * & * & * & -\tilde{R}
\end{array}\right)<0
$$


where

$$
\begin{aligned}
& (1,1)=\tilde{P} A^{T}+A \tilde{P}+X+X^{T}-2 \alpha \tilde{P} \\
& (1,3)=B \tilde{\Lambda}+k \tilde{P} C^{T} \\
& (1,4)=r_{M}\left(\tilde{P} A^{T}+X^{T}\right) \\
& (2,4)=r_{M} Y^{T} \\
& (3,4)=r_{M} \tilde{\Lambda} B^{T} .
\end{aligned}
$$

Moreover, the controller gains of (3) are given by $K=X \tilde{P}^{-1}$ and $L H=-M=-Y \tilde{R}^{-1}$, respectively.

Remark 6: It should be pointed out that even though we can derive $M=Y \tilde{R}^{-1}$ using (21), we can not guarantee to have the controller gain $L$ from $L H=-M$, which means that once $M$ is obtained, one should solve the equation $L H=-M$ to derive $L$. In some situations, in order to guarantee that there exists a solution $L$ to the equation $L H=-M$, we can set the matrix $M$ in the special structure depending on the information regarding matrices $L$ and $H$.

Remark 7: Different from a nonlinear optimization problem proposed by Suykens and Vandewalle [16], which is a nonconvex optimization problem, one can clearly see that the problem for designing the controller gains can be solved by an efficient convex optimization algorithm, i.e., an LMI solver feasp, which is well developed in Matlab LMI Toolbox [6]. It should be pointed out that although the nonlinear optimization problem in [16] is a nonconvex optimization problem, one can have convex subproblems depending on how one solves the optimization problem.

We need the following lemma to prove Proposition 4.

Lemma 2: For any real matrix $W \in \mathbb{R}^{n \times n}, W=W^{T}>0$, a nonsingular matrix $U \in \mathbb{R}^{n \times n}$, and a scalar $\mu>0$, then

$$
-U^{-1} W\left(U^{-1}\right)^{T} \leq \mu^{2} W^{-1}-\mu U^{-1}-\mu\left(U^{-1}\right)^{T}
$$

Proof: It is easy to see that

$$
\begin{aligned}
& \left(U^{-1} W\left(U^{-1}\right)^{T}+\mu^{2} W^{-1}\right)-\left(\mu U^{-1}+\mu\left(U^{-1}\right)^{T}\right) \\
& \quad=\left(U^{-1} W-\mu I\right) W^{-1}\left(U^{-1} W-\mu I\right)^{T} \\
& \quad \geq 0 .
\end{aligned}
$$

Re-arranging some terms yields (22). This completes the proof.

Proof of Proposition 4: Pre- and post-multiply both sides of (12) with $\operatorname{diag}\left(P^{-1}, R^{-1}, \Lambda^{-1}, R^{-1}\right)$, respectively, to obtain

$$
\left(\begin{array}{cccc}
(1,1) & M R^{-1}+P^{-1} & (1,3) & (1,4) \\
* & -R^{-1} & 0 & (2,4) \\
* & * & -2 \Lambda^{-1} & (3,4) \\
* & * & * & -R^{-1}
\end{array}\right)<0
$$

where

$$
\begin{aligned}
& (1,1)=P^{-1}(A+K)^{T}+(A+K) P^{-1}-P^{-1} R P^{-1} \\
& (1,3)=B \Lambda^{-1}+k P^{-1} C^{T} \\
& (1,4)=r_{M} P^{-1}(A+K)^{T} \\
& (2,4)=r_{M} R^{-1} M^{T} \\
& (3,4)=r_{M} \Lambda^{-1} B^{T} .
\end{aligned}
$$

For a given scalar $\alpha>0$, by Lemma 2, we have

$$
-P^{-1} R P^{-1} \leq \alpha^{2} R^{-1}-2 \alpha P^{-1} .
$$

Then, introducing new variables $\tilde{P}=P^{-1}, \tilde{R}=R^{-1}, \tilde{\Lambda}=$ $\Lambda^{-1}, X=K P^{-1}, Y=M R^{-1}$ and using Schur complement yield (21). This completes the proof.

Based on Proposition 2, the controller can be designed for three different situations: 1) $r_{d}<1$;2) $r_{d}=1$; 3) $r_{d}>1$.

Proposition 5: Under Case 2 with $r_{d}<1$, for given scalars $\alpha>0$ and $\beta>0$, the system described by (1)-(3) synchronizes, with the error system described by (5)-(7) having a unique and globally asymptotically stable equilibrium point $e(t)=0$, if there exist $n \times n$ real matrices $\tilde{P}=\tilde{P}^{T}>0, \tilde{Q}=\tilde{Q}^{T}>0$, $\tilde{R}=\tilde{R}^{T}>0$, and $\tilde{\Lambda}=\operatorname{diag}\left(\tilde{\lambda}_{1}, \tilde{\lambda}_{2}, \cdots, \tilde{\lambda}_{m}\right)>0, X, Y$ of appropriate dimensions such that

$$
\left(\begin{array}{ccccccc}
(1,1) & (1,2) & (1,3) & (1,4) & \alpha \tilde{R} & 0 & \tilde{P} \\
* & (2,2) & 0 & (2,4) & 0 & \beta \tilde{Q} & 0 \\
* & * & -2 \tilde{\Lambda} & (3,4) & 0 & 0 & 0 \\
* & * & * & -\tilde{R} & 0 & 0 & 0 \\
* & * & * & * & -\tilde{R} & 0 & 0 \\
* & * & * & * & * & (6,6) & 0 \\
* & * & * & * & * & * & -\tilde{Q}
\end{array}\right)<0
$$

where

$$
\begin{aligned}
& (1,1)=\tilde{P} A^{T}+A \tilde{P}+X+X^{T}-2 \alpha \tilde{P} \\
& (1,2)=Y+\tilde{P} \\
& (1,3)=B \tilde{\Lambda}+k \tilde{P} C^{T} \\
& (1,4)=r_{M}\left(\tilde{P} A^{T}+X^{T}\right) \\
& (2,2)=-(1+2 \beta) \tilde{R} \\
& (2,4)=r_{M} Y^{T} \\
& (3,4)=r_{M} \tilde{\Lambda} B^{T} \\
& (6,6)=-\left(1-r_{d}\right) \tilde{Q} .
\end{aligned}
$$

Moreover, the controller gains of (3) are given by $K=X \tilde{P}^{-1}$ and $L H=-M=-Y \tilde{R}^{-1}$, respectively.

Proof: Pre- and post-multiplying both sides of (14) with $\operatorname{diag}\left(P^{-1}, R^{-1}, \Lambda^{-1}, R^{-1}\right)$, respectively, we have

$$
\left(\begin{array}{cccc}
(1,1) & M R^{-1}+P^{-1} & (1,3) & (1,4) \\
* & (2,2) & 0 & (2,4) \\
* & * & -2 \Lambda^{-1} & (3,4) \\
* & * & * & -R^{-1}
\end{array}\right)<0
$$

where

$$
\begin{aligned}
(1,1)= & P^{-1}(A+K)^{T}+(A+K) P^{-1} \\
& +P^{-1} Q P^{-1}-P^{-1} R P^{-1} \\
(1,3)= & B \Lambda^{-1}+k P^{-1} C^{T} \\
(1,4)= & r_{M} P^{-1}(A+K)^{T} \\
(2,2)= & -\left(1-r_{d}\right) R^{-1} Q R^{-1}-R^{-1} \\
(2,4)= & r_{M} R^{-1} M^{T} \\
(3,4)= & r_{M} \Lambda^{-1} B^{T} .
\end{aligned}
$$


Notice that (23), and for a given scalar $\beta>0$, use Lemma 2 to obtain

$$
-R^{-1}\left[\left(1-r_{d}\right) Q\right] R^{-1} \leq \beta^{2}\left[\left(1-r_{d}\right) Q\right]^{-1}-2 \beta R^{-1} .
$$

Then, introducing new variables $\tilde{P}=P^{-1}, \tilde{Q}=Q^{-1}, \tilde{R}=$ $R^{-1}, \tilde{\Lambda}=\Lambda^{-1}, X=K P^{-1}, Y=M R^{-1}$ and using Schur complement yield (24). This completes the proof.

Remark 8: It should be pointed out that instead of using Lemma 2 to handle the nonlinear terms $-P^{-1} R P^{-1}$ and $-R^{-1} Q R^{-1}$ in (25) in the proof of Proposition 5, one can introduce two new variables $S_{1}$ and $S_{2}$ such that

$$
-P^{-1} R P^{-1}<-S_{1}^{-1}, \quad-R^{-1} Q R^{-1}<-S_{2}^{-1}
$$

which are equivalent to

$$
\left(\begin{array}{cc}
-S_{1} & P \\
P & -R
\end{array}\right)<0, \quad\left(\begin{array}{cc}
-S_{2} & R \\
R & -Q
\end{array}\right)<0 .
$$

Then, (25) becomes

$$
\left(\begin{array}{cccc}
(1,1) & M R^{-1}+P^{-1} & (1,3) & (1,4) \\
* & (2,2) & 0 & (2,4) \\
* & * & -2 \Lambda^{-1} & (3,4) \\
* & * & * & -R^{-1}
\end{array}\right)<0
$$

where

$$
\begin{aligned}
& (1,1)=P^{-1}(A+K)^{T}+(A+K) P^{-1}+P^{-1} Q P^{-1}-S_{1}^{-1} \\
& (1,3)=B \Lambda^{-1}+k P^{-1} C^{T} \\
& (1,4)=r_{M} P^{-1}(A+K)^{T} \\
& (2,2)=-\left(1-r_{d}\right) S_{2}^{-1}-R^{-1} \\
& (2,4)=r_{M} R^{-1} M^{T} \\
& (3,4)=r_{M} \Lambda^{-1} B^{T}
\end{aligned}
$$

Introduce new variables $\tilde{P}=P^{-1}, \tilde{Q}=Q^{-1}, \tilde{R}=R^{-1}$, $\tilde{\Lambda}=\Lambda^{-1}, X=K P^{-1}, Y=M R^{-1}, \tilde{S}_{1}=S_{1}^{-1}, \tilde{S}_{2}=S_{2}^{-1}$ and use Schur complement to obtain

$$
\left(\begin{array}{ccccc}
(1,1) & Y+\tilde{P} & (1,3) & (1,4) & \tilde{P} \\
* & (2,2) & 0 & (2,4) & 0 \\
* & * & -2 \tilde{\Lambda} & (3,4) & 0 \\
* & * & * & -\tilde{R} & 0 \\
* & * & * & * & -\tilde{Q}
\end{array}\right)<0
$$

where

$$
\begin{aligned}
& (1,1)=\tilde{P} A^{T}+A \tilde{P}+X+X^{T}-\tilde{S}_{1} \\
& (1,4)=r_{M}\left(\tilde{P} A^{T}+X^{T}\right) \\
& (2,2)=-\left(1-r_{d}\right) \tilde{S}_{2}-\tilde{R} \\
& (2,4)=r_{M} Y^{T} \\
& (3,4)=r_{M} \tilde{\Lambda} B^{T} .
\end{aligned}
$$

Then, we can formulate a minimization problem as

$$
\text { Minimize } \operatorname{tr}\left(P \tilde{P}+Q \tilde{Q}+R \tilde{R}+S_{1} \tilde{S}_{1}+S_{2} \tilde{S}_{2}\right)
$$$$
\text { subjectto }(26),(28) \text {, and }
$$

$$
\left\{\begin{array}{l}
\left(\begin{array}{cc}
P & I \\
I & \tilde{P}
\end{array}\right) \geq 0,\left(\begin{array}{cc}
Q & I \\
I & \tilde{Q}
\end{array}\right) \geq 0 \\
\left(\begin{array}{cc}
R & I \\
I & \tilde{R}
\end{array}\right) \geq 0,\left(\begin{array}{cc}
S_{1} & I \\
I & \tilde{S}_{1}
\end{array}\right) \geq 0 \\
\left(\begin{array}{cc}
S_{2} & I \\
I & \tilde{S}_{2}
\end{array}\right) \geq 0
\end{array}\right.
$$

If $\operatorname{tr}\left(P \tilde{P}+Q \tilde{Q}+R \tilde{R}+S_{1} \tilde{S}_{1}+S_{2} \tilde{S}_{2}\right)=5 n$, we can conclude that under Case 2 with $r_{d}<1$, the system described by (1)-(3) synchronizes and the controller gains of (3) are given by $K=$ $X \tilde{P}^{-1}$ and $L H=-M=-Y \tilde{R}^{-1}$, respectively.

Proposition 6: Under Case 2 with $r_{d}=1$, for given scalar $\alpha>0$, the system described by (1)-(3) synchronizes, with the error system described by (5)-(7) having a unique and globally asymptotically stable equilibrium point $e(t)=0$, if there exist $n \times n$ real matrices $\tilde{P}=\tilde{P}^{T}>0, \tilde{Q}=\tilde{Q}^{T}>0, \tilde{R}=\tilde{R}^{T}>$ 0 , and $\tilde{\Lambda}=\operatorname{diag}\left(\tilde{\lambda}_{1}, \tilde{\lambda}_{2}, \cdots, \tilde{\lambda}_{m}\right)>0, X, Y$ of appropriate dimensions such that

$$
\left(\begin{array}{cccccc}
(1,1) & (1,2) & (1,3) & (1,4) & \alpha \tilde{R} & \tilde{P} \\
* & -\tilde{R} & 0 & (2,4) & 0 & 0 \\
* & * & -2 \tilde{\Lambda} & (3,4) & 0 & 0 \\
* & * & * & -\tilde{R} & 0 & 0 \\
* & * & * & * & -\tilde{R} & 0 \\
* & * & * & * & * & -\tilde{Q}
\end{array}\right)<0
$$

where

$$
\begin{aligned}
& (1,1)=\tilde{P} A^{T}+A \tilde{P}+X+X^{T}-2 \alpha \tilde{P} \\
& (1,2)=Y+\tilde{P} \\
& (1,3)=B \tilde{\Lambda}+k \tilde{P} C^{T} \\
& (1,4)=r_{M}\left(\tilde{P} A^{T}+X^{T}\right) \\
& (2,4)=r_{M} Y^{T} \\
& (3,4)=r_{M} \tilde{\Lambda} B^{T} .
\end{aligned}
$$

Moreover, the controller gains of (3) are given by $K=X \tilde{P}^{-1}$ and $L H=-M=-Y \tilde{R}^{-1}$, respectively.

Proof: Notice that for $r_{d}=1$, we have $-\left(1-r_{d}\right) Q=0$. The remaining proof is the same as that in Proposition 5. This completes the proof.

Proposition 7: Under Case 2 with $r_{d}>1$, for a given scalar $\alpha>0$, the system described by (1)-(3) synchronizes, with the error system described by (5)-(7) having a unique and globally asymptotically stable equilibrium point $e(t)=0$, if there exist real matrices $\tilde{P}=\tilde{P}^{T}>0, \tilde{Q}=\tilde{Q}^{T}>0, \tilde{R}=\tilde{R}^{T}>$ 0 , and $\tilde{\Lambda}=\operatorname{diag}\left(\tilde{\lambda}_{1}, \tilde{\lambda}_{2}, \cdots, \tilde{\lambda}_{m}\right)>0, X, Y$ of appropriate dimensions such that

$$
\left(\begin{array}{ccccccc}
(1,1) & (1,2) & (1,3) & (1,4) & \alpha \tilde{R} & 0 & \tilde{P} \\
* & -\tilde{R} & 0 & (2,4) & 0 & (2,6) & 0 \\
* & * & -2 \tilde{\Lambda} & (3,4) & 0 & 0 & 0 \\
* & * & * & -\tilde{R} & 0 & 0 & 0 \\
* & * & * & * & -\tilde{R} & 0 & 0 \\
* & * & * & * & * & (6,6) & 0 \\
* & * & * & * & * & * & -\tilde{Q}
\end{array}\right)<0
$$


TABLE I

The MAXIMUM Allowed TIME-DELAY BOUND $r_{M}$ FOR DIFFERENT $r_{d}$

\begin{tabular}{c|c|c|c|c|c|c}
\hline$r_{d}$ & 0 & 0.3 & 0.6 & 0.9 & 1.0 & $>1.0$ \\
\hline$[11]$ & 0.1121 & 0.0814 & 0.0487 & 0.0130 & - & - \\
\hline Prop 1 & 0.1527 & 0.1527 & 0.1527 & 0.1527 & 0.1527 & 0.1527 \\
\hline Prop 2 & 0.1622 & 0.1591 & 0.1566 & 0.1541 & 0.1527 & 0.1527 \\
\hline
\end{tabular}

where

$$
\begin{aligned}
& (1,1)=\tilde{P} A^{T}+A \tilde{P}+X+X^{T}-2 \alpha \tilde{P} \\
& (1,2)=Y+\tilde{P} \\
& (1,3)=B \tilde{\Lambda}+k \tilde{P} C^{T} \\
& (1,4)=r_{M}\left(\tilde{P} A^{T}+X^{T}\right) \\
& (2,4)=r_{M} Y^{T} \\
& (2,6)=\left(r_{d}-1\right) \tilde{R} \\
& (3,4)=r_{M} \tilde{\Lambda} B^{T} \\
& (6,6)=-\left(r_{d}-1\right) \tilde{Q} .
\end{aligned}
$$

Moreover, the controller gains of (3) are given by $K=X \tilde{P}^{-1}$ and $L H=-M=-Y \tilde{R}^{-1}$, respectively.

Proof: Notice that $-\left(1-r_{d}\right) R^{-1} Q R^{-1}>0$ for $r_{d}>$ 1. Apply Schur complement to $-\left(1-r_{d}\right) R^{-1} Q R^{-1}$ in $(2,2)$ block in (25), and follow the same proof in Proposition 5 for the remaining part. This completes the proof.

\section{EXAMPLE}

In order to show the effectiveness of the derived results in this paper, we consider the following Chua's Circuit

$$
\left\{\begin{array}{l}
\dot{x}=\alpha(y-h(x)) \\
\dot{y}=x-y+z \\
\dot{z}=-\beta y
\end{array}\right.
$$

with nonlinear characteristic

$$
h(x)=m_{1} x+\frac{1}{2}\left(m_{0}-m_{1}\right)(|x+c|-|x-c|)
$$

and parameters $m_{0}=-(1 / 7), m_{1}=(2 / 7), \alpha=9, \beta=14.28$, and $c=1$ (Chua et al. [3]; Madan [15]). The system can be represented in Lur'e form [23] with

$$
\begin{aligned}
& A=\left(\begin{array}{ccc}
-\alpha m_{1} & \alpha & 0 \\
1 & -1 & 1 \\
0 & -\beta & 0
\end{array}\right), \quad B=\left(\begin{array}{c}
-\alpha\left(m_{0}-m_{1}\right) \\
0 \\
0
\end{array}\right) \\
& C=H=\left(\begin{array}{lll}
1 & 0 & 0
\end{array}\right)
\end{aligned}
$$

and $\varphi(\xi)=(1 / 2)(|\xi+c|-|\xi-c|)$ belonging to sector $[0, k]$ with $k=1$.

We first consider the stability analysis. In order to compare with the result in [11] and show the effectiveness of Propositions 1 and 2 , let the controller gains be]

$$
K=\left(\begin{array}{ccc}
-1 & 0 & 0 \\
0 & -1 & 0 \\
0 & 0 & -1
\end{array}\right), \quad L=\left(\begin{array}{c}
6.0229 \\
1.3367 \\
-2.1264
\end{array}\right)
$$

Applying the criterion (Theorem 1) in [11] and Propositions 1 and 2, the maximum allowed delay bound is listed in Table I,
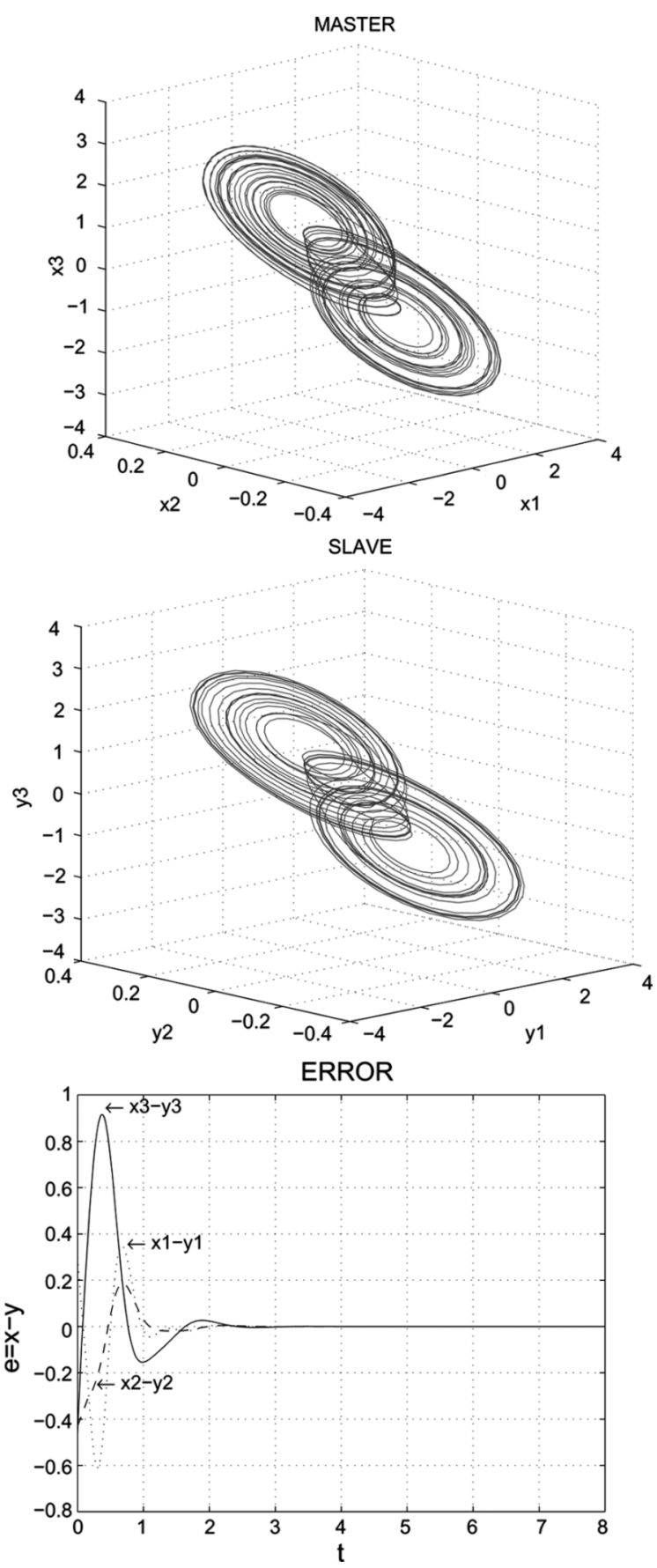

Fig. 1. Simulation results for master, slave, and error systems for delay $r_{M}=$ 0.1527 .

from which one can clearly see that for $r_{d}<1$, both Proposition 1 and Proposition 2 can provide a larger bound than the criterion in [11]; for $r_{d} \geq 1$, the criterion in [11] fails to make any conclusion while Proposition 1 and Proposition 2 are still valid to give the results. From the table, one can also see that for $r_{d}<1$, one can obtain better results using Proposition 2 than Proposition 1; for $r_{d} \geq 1$, the same results are derived using Propositions 1 and 2, which further verifies Proposition 3 through this example. Figs. 1 and 2 give the simulation results for master, slave and error systems for delay $r=0.1527$ and 0.1622 , respectively. One can clearly see that the master and slave systems are indeed synchronized. 

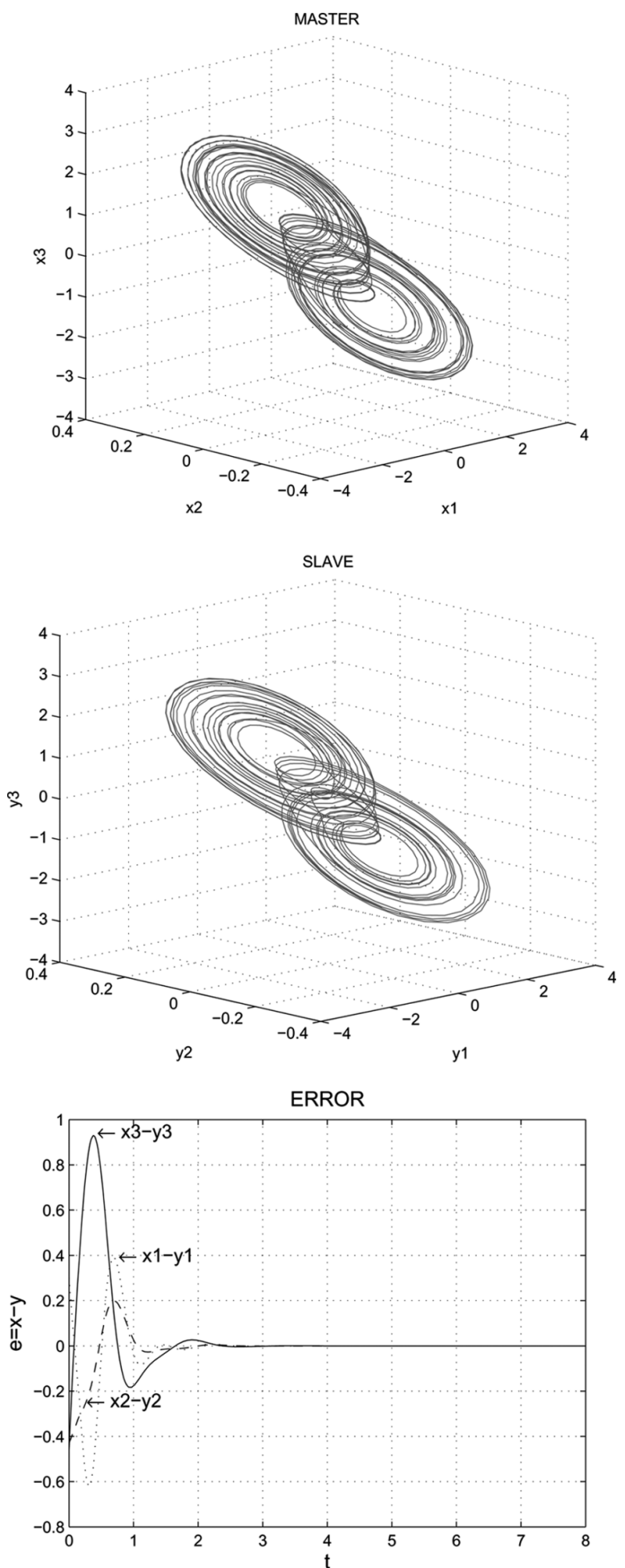

Fig. 2. Simulation results for master, slave, and error systems for delay $r_{M}=$ 0.1622 .

Next, we address the controller design.

Case 1) Let $r_{M}=0.8$ and choose $\alpha=0.1$. Using Proposition 4 , we have

$$
\begin{aligned}
P & =\left(\begin{array}{ccc}
0.3326 & -0.0285 & -0.0536 \\
-0.0285 & 1.3329 & -0.0661 \\
-0.0536 & -0.0661 & 0.8447
\end{array}\right) \\
R & =\left(\begin{array}{ccc}
2.0207 & 0 & 0 \\
0 & 3.1534 & 0 \\
0 & 0 & 3.3215
\end{array}\right)
\end{aligned}
$$

$$
\begin{aligned}
X & =\left(\begin{array}{ccc}
-0.7974 & -11.8961 & 0.8672 \\
-0.4126 & -0.0967 & -1.2968 \\
-0.7089 & 18.6310 & -3.2381
\end{array}\right) \\
Y & =\left(\begin{array}{ccc}
-0.0661 & 0 & 0 \\
0.8447 & 0 & 0 \\
2.0207 & 0 & 0
\end{array}\right), \quad \lambda=0.1461 .
\end{aligned}
$$

Then, the feedback gains are given by

$$
\begin{aligned}
K & =\left(\begin{array}{ccc}
-3.1486 & -8.9861 & 0.1236 \\
-1.5222 & -0.1867 & -1.6465 \\
-1.4058 & 13.8066 & -2.8424
\end{array}\right) \\
L & =\left(\begin{array}{c}
0.0327 \\
-0.4180 \\
-1.0000
\end{array}\right)
\end{aligned}
$$

We give the simulation results for master, slave and error systems for the above derived gains and delay $r_{M}=0.8$ in Fig. 3, from which one can see that the master and slave systems are synchronized.

Case 2) (i) For $r_{M}=0.9, r_{d}=0.5<1$, choosing $\alpha=0.1$ and $\beta=0.6$, applying Proposition 5 yield

$$
\begin{aligned}
& P=\left(\begin{array}{ccc}
0.1042 & -0.0047 & 0.0001 \\
-0.0047 & 0.3000 & -0.0002 \\
0.0001 & -0.0002 & 0.2483
\end{array}\right) \\
& Q=\left(\begin{array}{ccc}
0.5497 & -0.0128 & 0.0002 \\
-0.0128 & 0.8476 & 0.0000 \\
0.0002 & 0.0000 & 0.8290
\end{array}\right) \\
& R=\left(\begin{array}{ccc}
0.8391 & 0 & 0 \\
0 & 0.8125 & 0 \\
0 & 0 & 0.7703
\end{array}\right) \\
& X=\left(\begin{array}{ccc}
-0.2165 & -2.7443 & 0.0026 \\
-0.1812 & -0.1757 & -0.2465 \\
-0.0654 & 4.2866 & -0.3678
\end{array}\right) \\
& Y=\left(\begin{array}{ccc}
0.2483 & 0 & 0 \\
0.5497 & 0 & 0 \\
-0.0128 & 0 & 0
\end{array}\right), \quad \lambda=0.0319 \text {. }
\end{aligned}
$$

Then, the feedback gains are given by

$$
\begin{aligned}
K & =\left(\begin{array}{ccc}
-2.4920 & -9.1862 & 0.0057 \\
-1.7648 & -0.6137 & -0.9920 \\
0.0190 & 14.2875 & -1.4717
\end{array}\right) \\
L & =\left(\begin{array}{c}
-0.2959 \\
-0.6551 \\
0.0153
\end{array}\right) .
\end{aligned}
$$

(ii) For $r_{M}=0.8, r_{d}=1$, choose $\alpha=0.1$ and use Proposition 6 to obtain

$$
\begin{aligned}
& P=\left(\begin{array}{ccc}
0.1357 & -0.0095 & 0.0001 \\
-0.0095 & 0.3815 & 0.0000 \\
0.0001 & 0.0000 & 0.3109
\end{array}\right) \\
& Q=\left(\begin{array}{ccc}
0.6195 & -0.0048 & 0.0000 \\
-0.0048 & 1.1143 & 0.0000 \\
0.0000 & 0.0000 & 1.0891
\end{array}\right)
\end{aligned}
$$



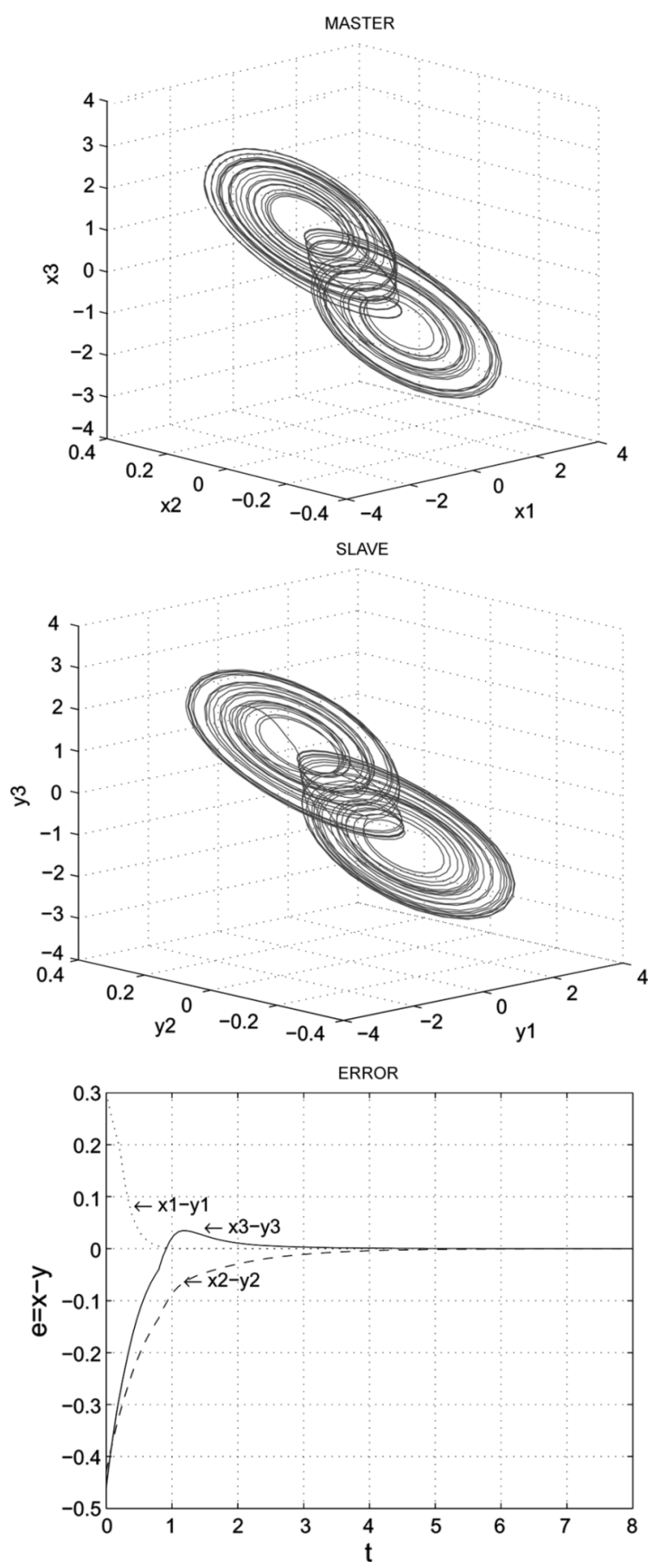

Fig. 3. Simulation results for master, slave and error systems for Case I.

$$
\begin{aligned}
R & =\left(\begin{array}{ccc}
1.3058 & 0 & 0 \\
0 & 1.1852 & 0 \\
0 & 0 & 1.1272
\end{array}\right) \\
X & =\left(\begin{array}{ccc}
-0.4132 & -3.5046 & 0.0008 \\
-0.2541 & -0.2809 & -0.3101 \\
-0.1352 & 5.4484 & -0.5014
\end{array}\right) \\
Y & =\left(\begin{array}{ccc}
0.3109 & 0 & 0 \\
0.6195 & 0 & 0 \\
-0.0048 & 0 & 0
\end{array}\right), \quad \lambda=0.0549 .
\end{aligned}
$$
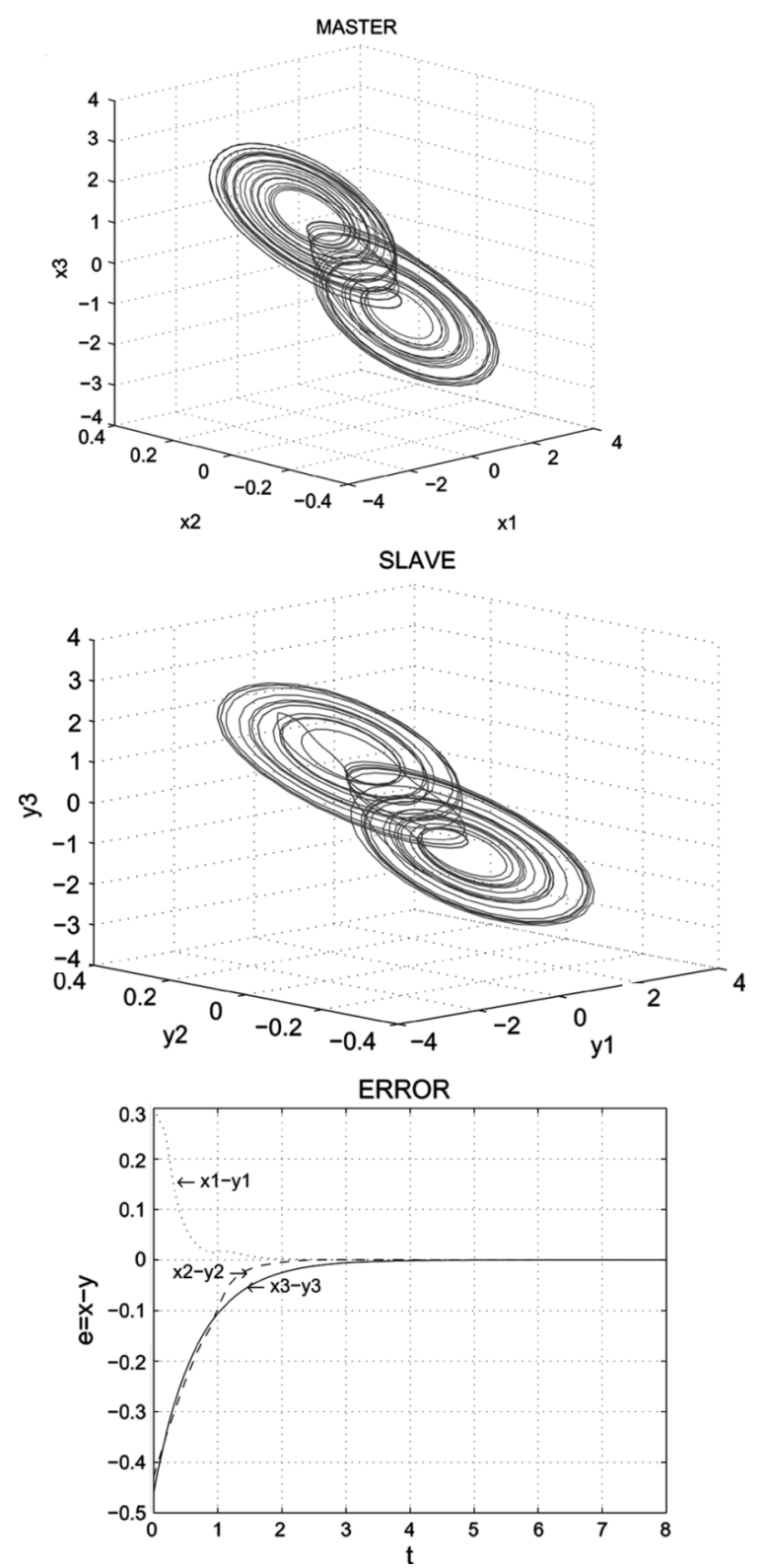

Fig. 4. Simulation results for master, slave and error systems for Case II-(i).

Then, we derive the feedback gains as

$$
\begin{aligned}
K & =\left(\begin{array}{ccc}
-3.6981 & -9.2800 & 0.0025 \\
-1.9271 & -0.7846 & -0.9970 \\
0.0080 & 14.2834 & -1.6112
\end{array}\right) \\
L & =\left(\begin{array}{c}
-0.2381 \\
-0.4744 \\
0.0037
\end{array}\right) .
\end{aligned}
$$

(iii) For $r_{M}=0.8, r_{d}=1.5>1$, choosing $\alpha=0.1$ and employing Proposition 7, we have

$$
\begin{aligned}
P & =\left(\begin{array}{ccc}
0.3139 & -0.0887 & -0.0005 \\
-0.0887 & 1.1399 & 0.0014 \\
-0.0005 & 0.0014 & 0.4661
\end{array}\right) \\
Q & =\left(\begin{array}{lll}
1.8419 & 0.0493 & 0.0001 \\
0.0493 & 4.4303 & 0.0000 \\
0.0001 & 0.0000 & 4.0150
\end{array}\right)
\end{aligned}
$$



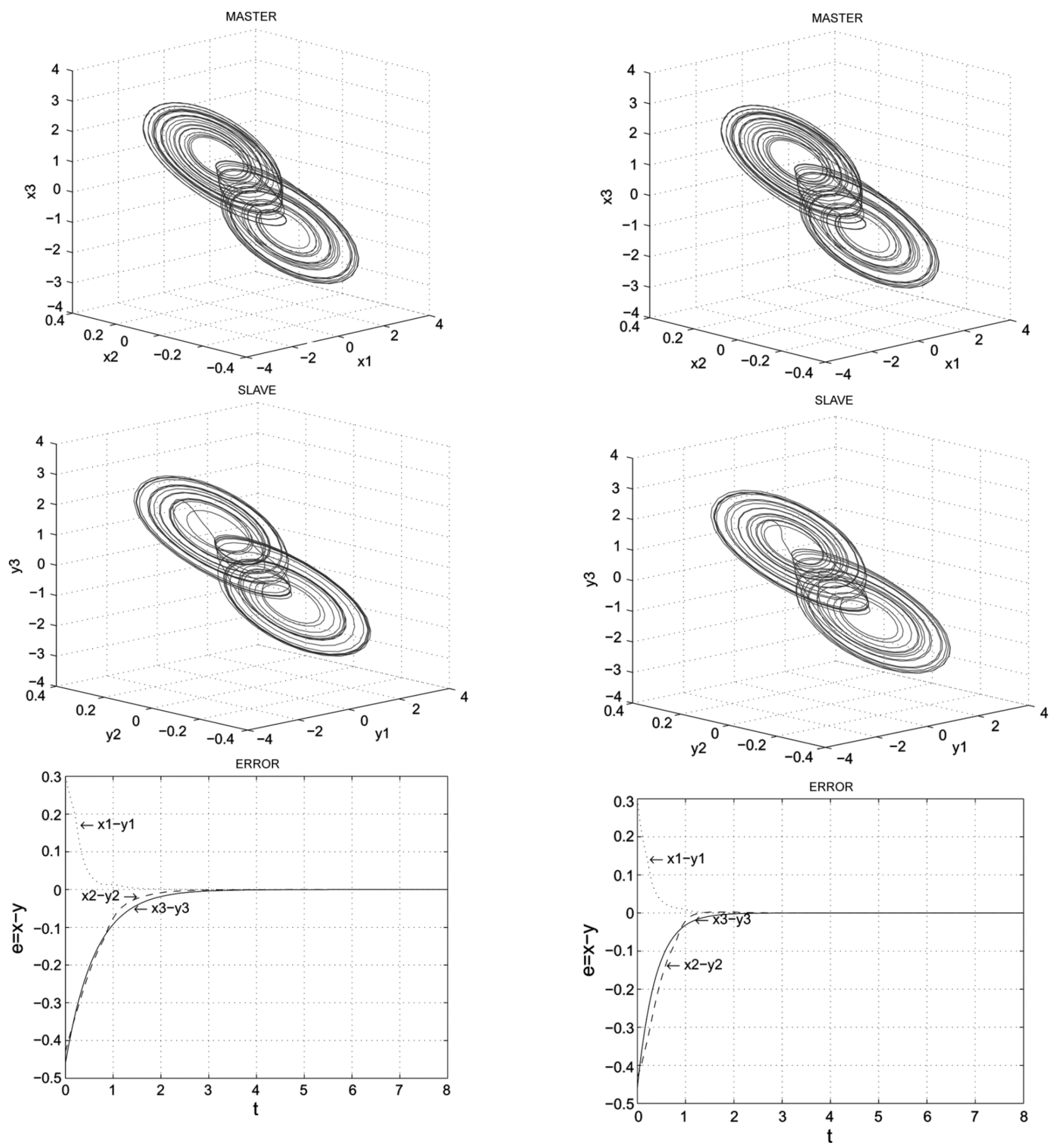

Fig. 5. Simulation results for master, slave and error systems for Case II-(ii)

Fig. 6. Simulation results for master, slave and error systems for Case II-(iii).

$$
\begin{aligned}
R & =\left(\begin{array}{ccc}
2.1258 & 0 & 0 \\
0 & 3.6648 & 0 \\
0 & 0 & 2.5755
\end{array}\right) \\
X & =\left(\begin{array}{ccc}
-1.2983 & -10.8617 & -0.0220 \\
-1.3801 & -2.2061 & -0.4905 \\
-1.2936 & 16.2313 & -1.2057
\end{array}\right) \\
Y & =\left(\begin{array}{lll}
0.4661 & 0 & 0 \\
1.8419 & 0 & 0 \\
0.0493 & 0 & 0
\end{array}\right), \quad \lambda=0.1785 .
\end{aligned}
$$

One obtains the feedback gains as

$$
\begin{aligned}
K & =\left(\begin{array}{lll}
-6.9841 & -10.0723 & -0.0260 \\
-5.0569 & -2.3277 & -1.0515 \\
-0.1016 & 14.2344 & -2.6284
\end{array}\right) \\
L & =\left(\begin{array}{l}
-0.2192 \\
-0.8664 \\
-0.0232
\end{array}\right) .
\end{aligned}
$$

The simulation results for master, slave and error systems for the above derived gains and delays for 
Case II (i)-(iii) are illustrated in Figs. 4-6, respectively. From these simulation results, one can clearly see that the master and slave systems are synchronized, which means that the design method is effective.

\section{CONCLUSION}

The problem of designing time-varying delay feedback controllers for master-slave synchronization of Lur'e systems has been addressed. Some delay-dependent synchronization criteria have been obtained. In order to reduce the conservatism of the criteria, we have avoided using model transformation and bound technique for cross terms, which are used in the literature in deriving delay-dependent synchronization criteria for Lur'e systems. We have successfully built the relationship between the criteria for the two cases of time-varying delays and have concluded that if the time-varying delay is differentiable and the bound of the time derivative of the time-varying delay is less than one, we can derive a less conservative result using the criterion for the second case than that for the first case; on the other hand, when the bound of the time derivative of the time-varying delay is equal to or greater than one, we can get the same results using the criteria for the first case or second case; however, if the time-varying delay is not differentiable, only the criterion for the first case can be used to handle the situation. Based on the newly-established synchronization criteria, we have derived sufficient conditions on the existence of a time-varying delay feedback controller. Based on these sufficient conditions, we have designed controller gains by solving a set of LMIs. We have also illustrated the effectiveness of the design method through Chua's circuit.

\section{ACKNOWLEDGMENT}

The author would like to thank Mr. D. Han for numerical example simulations.

\section{REFERENCES}

[1] J. Cao, H. X. Li, and D. W. C. Ho, "Synchronization criteria of Lur'e systems with time-delay feedback control," Chaos, Solitons, Fractals, vol. 23, no. 4, pp. 1285-1298, 2005.

[2] G. Chen and X. Dong, From Chaos to Order-Perspectives, Methodologies, and Applications. Singapore: World Scientific, 1998.

[3] L. O. Chua, M. Komura, and T. Matsumoto, "The double scroll family," IEEE Trans. Circuits Syst. I, Fundam. Theory Appl., vol. CAS-33, no. 11, pp. 1072-1118, Nov. 1986.

[4] P. F. Curran and L. O. Chua, "Absolute stability theory and the synchronization problem," Int. J. Bifurc. Chaos, vol. 7, no. 6, pp. 1375-1383, 1997.

[5] P. F. Curran, J. A. K. Suykens, and L. O. Chua, "Absolute stability theory and master-slave synchronization problem," Int. J. Bifurc. Chaos, vol. 7, no. 12, pp. 2891-2896, 1997.

[6] P. Gahinet, A. Nemirovski, A. J. Laub, and M. Chilali, LMI Control Toolbox-For Use With MATLAB. Natick: The MathWorks, Inc., 1995.

[7] K. Gu, V. L. Kharitonov, and J. Chen, Stability of Time-Delay Systems. Boston, MA: Birkhäuser, 2003.

[8] Q.-L. Han, "Robust stability of uncertain delay-differential systems of neutral type," Automatica, vol. 38, no. 4, pp. 719-723, 2002.
[9] Q.-L. Han, "Stability criteria for a class of linear neutral systems with time-varying discrete and distributed delays," IMA J. Math. Contr. Inf., vol. 20, no. 4, pp. 371-386, 2003.

[10] M. Hasler, "Synchronization principles and applications," in Proc. Circuits Syst.: Tutorials IEEE-ISCAS'94, 1994, pp. 314-326.

[11] H. Huang, H. X. Li, and J. Zhong, "Master-slave synchronization of general Lur'e systems with time-varying delay and parameter uncertainty," Int. J. Bifurc. Chaos, vol. 16, no. 2, pp. 281-294, 2006.

[12] L. Kocarev, K. S. Halle, K. Eckert, L. O. Chua, and A. Shang, "Experimental demonstration of secure communications via chaotic synchronization," Int. J. Bifurc. Chaos, vol. 2, no. 3, pp. 709-713, 1992.

[13] X. Liao and G. Chen, "Chaos synchronization of general Lur'e systems via time-delay feedback control," Int. J. Bifurc. Chaos, vol. 13, no. 1, pp. 207-213, 2003.

[14] X. Liao, G. Chen, B. Xu, and Y. Shen, "On global exponential synchronization of Chua circuits," Int. J. Bifurc. Chaos, vol. 15, no. 7, pp. 2227-2234, 2005.

[15] R. N. Madan, Chua Circuit: A Paradigm for Chaos. Singapore: World Scientific, 1993.

[16] J. A. K. Suykens and J. Vandewalle, "Master-slave synchronization of Lur'e systems," Int. J. Bifurc. Chaos, vol. 7, no. 3, pp. 665-669, 1997.

[17] J. A. K. Suykens, P. F. Curran, and L. O. Chua, "Master-slave synchronization using dynamic output feedback," Int. J. Bifurc. Chaos, vol. 7, no. 3, pp. 671-679, 1997.

[18] J. A. K. Suykens, P. F. Curran, T. Yang, J. Vandewalle, and L. O. Chua, "Nonlinear $H_{\infty}$ synchronization of Lur'e systems: Dynamic output feedback case," IEEE Trans. Circuits Syst. I, Fundam. Theory Appl., vol. 44, no. 11, pp. 1089-1092, Nov. 1997.

[19] J. A. K. Suykens, P. F. Curran, and L. O. Chua, "Robust synthesis for master-slave synchronization of Lur'e systems," IEEE Trans. Circuits Syst. I, Fundam. Theory Appl., vol. 46, no. 7, pp. 841-850, Jul. 1999.

[20] X. Wu, J. P. Cai, and Y.Zhao, "Revision and improvement of a theorem for robust synchronization of nonidentical Lur'e systems," IEEE Trans. Circuits Syst. II, Exp. Briefs, vol. 52, no. 7, pp. 429-432, Jul. 2005.

[21] X. Wu and Y. Zhao, "Frequency domain criterion for chaos synchronization of Lur'e systems via linear state error feedback control," Int. J. Bifurc. Chaos, vol. 15, no. 4, pp. 1445-1454, 2005.

[22] C. W. Wu and L. O. Chua, "A unified framework for synchronization and control of dynamical systems," Int. J. Bifurc. Chaos, vol. 4, no. 4, pp. 979-989, 1994.

[23] M. E. Yalçin, J. A. K. Suykens, and J. Vandewalle, "Master-slave synchronization of Lur'e systems with time-delay," Int. J. Bifurc. Chaos, vol. 11, no. 6, pp. 1707-1722, 2001.

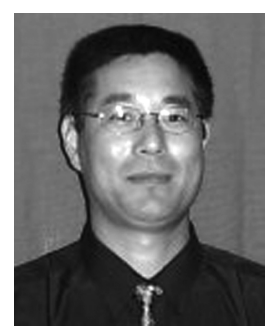

Qing-Long Han received the B.Sc. degree in mathematics from the Shandong Normal University, Jinan, China, in 1983, and the M.Eng. and Ph.D. degrees in information science (electrical engineering) from the East China University of Science and Technology, Shanghai, China, in 1992 and 1997, respectively.

From September 1997 to December 1998, he was a Post-Doctoral Researcher Fellow in LAII-ESIP, Université de Poitiers, France. From January 1999 to August 2001, he was a Research Assistant Professor in the Department of Mechanical and Industrial Engineering, Southern Illinois University, Edwardsville. In September 2001, he joined the Central Queensland University, Australia, where he is currently an Associate Professor in the School of Computing Sciences and an Associate Dean (Research and Innovation) in the Faculty of Business and Informatics. He has held a Visiting Professor position in LAII-ESIP, Université de Poitiers, France, a Chair Professor position in Hangzhou Dianzi University, China, as well as a Guest Professor position in three Chinese universities. His research interests include time-delay systems, robust control, networked control systems, neural networks, complex systems and software development processes He has published over 140 refereed papers in technical journals and conference proceedings.

Prof. Han has served as an Associate Editor for Dynamics of Continuous, Discrete \& Impulsive Systems-Series B: Applications \& Algorithms (2004Present), and a Guest Editor for the Special Issue on Time-Delay Systems in Asian Journal of Control (2004-2006), and a Guest Editor for the Special Issue on Time-Delay Systems in Dynamics of Continuous, Discrete and Impulsive Systems-Series B: Applications \& Algorithms (2006-present). 